PROCEEDINGS OF THE

AMERICAN MATHEMATICAL SOCIETY

Volume 136, Number 10, October 2008, Pages 3395-3404

S 0002-9939(08)09516-6

Article electronically published on June 10, 2008

\title{
ANALOGUES OF THE ARTIN FACTORIZATION FORMULA FOR THE AUTOMORPHIC SCATTERING MATRIX AND SELBERG ZETA-FUNCTION ASSOCIATED TO A KLEINIAN GROUP
}

\author{
JOSHUA S. FRIEDMAN
}

(Communicated by Wen-Ching Winnie Li)

\begin{abstract}
For Kleinian groups acting on a hyperbolic three-space, we prove factorization formulas for both the Selberg zeta-function and the automorphic scattering matrix. We extend results of Venkov and Zograf from Fuchsian groups to Kleinian groups, and we give a proof that is simple and extendable to more general groups.
\end{abstract}

\section{INTRODUCTION}

In VZ83, Venkov and Zograf gave an analogue of Artin's well-known factorization formula. More specifically, they gave factorization formulas for both the Selberg zeta-function and automorphic scattering matrix that are associated to a Fuchsian group (in the context of the Selberg spectral theory of automorphic functions).

Let $\Gamma<\operatorname{PSL}(2, \mathbb{C})$ be a cofinite Kleinian group, and let $\Gamma_{1}$ be a finite-index normal subgroup of index $n$. Let $\chi \in \operatorname{Rep}\left(\Gamma_{1}, V\right)$ be a finite-dimensional unitary representation of $\Gamma_{1}$ in $V$, and let $\psi=U^{\chi} \in \operatorname{Rep}\left(\Gamma, V^{n}\right)$ be its induced representation to $\Gamma$.

Let $\mathfrak{S}\left(s, \Gamma_{1}, \chi\right), \mathfrak{S}(s, \Gamma, \psi)$ be automorphic scattering matrices (defined in $\$ 5$ ), and set

$$
\begin{aligned}
\phi\left(s, \Gamma_{1}, \chi\right) & \equiv \operatorname{det} \mathfrak{S}\left(s, \Gamma_{1}, \chi\right), \\
\phi(s, \Gamma, \psi) & \equiv \operatorname{det} \mathfrak{S}(s, \Gamma, \psi) .
\end{aligned}
$$

Theorem. Let $\Gamma$ be a cofinite Kleinian group, and let $\Gamma_{1}$ be a finite-index normal subgroup of $\Gamma$. Let $\chi \in \operatorname{Rep}\left(\Gamma_{1}, V\right)$, and $\psi=U^{\chi}$. Then for all regular $s \in \mathbb{C}$,

$$
\phi\left(s, \Gamma_{1}, \chi\right)=\phi(s, \Gamma, \psi) .
$$

In VZ83 a slightly weaker version of this equation was derived. The authors showed that

$$
\phi\left(s, \Gamma_{1}, \chi\right) \Omega\left(\Gamma_{1}, \chi\right)^{1-2 s}=\phi\left(s, \Gamma, U^{\chi}\right) \Omega\left(\Gamma, U^{\chi}\right)^{1-2 s},
$$

where $\Omega(\cdot, \cdot)$ is a constant depending on the group and unitary representation.

We also have

Received by the editors March 2, 2007

2000 Mathematics Subject Classification. Primary 11F72.

(C)2008 American Mathematical Society Reverts to public domain 28 years from publication 
Theorem. Let $\Gamma$ be an arbitrary Kleinian group, and let $\Gamma_{1}$ be a finite-index normal subgroup of $\Gamma$. Let $\chi \in \operatorname{Rep}\left(\Gamma_{1}, V\right)$, and $\psi=U^{\chi}$. Then for $\operatorname{Re} s>1$,

$$
Z\left(s, \Gamma_{1}, \chi\right)=Z(s, \Gamma, \psi) .
$$

Here $Z(s, \cdot, \cdot)$ is the Selberg zeta-function (defined in $\$ 2.2$ ).

Theorem. Let $\Gamma$ be an arbitrary Kleinian group, and let $\Gamma_{1}$ be a finite-index normal subgroup of $\Gamma$. Then for $\operatorname{Re} s>1$,

$$
Z\left(s, \Gamma_{1}, \mathbf{1}\right)=Z\left(s, \Gamma, U^{\mathbf{1}}\right)=\prod_{\vartheta \in\left(\Gamma_{1} \backslash \Gamma\right)^{*}} Z(s, \Gamma, \vartheta)^{n_{\vartheta}} .
$$

Here $\left(\Gamma_{1} \backslash \Gamma\right)^{*}$ is the set of all pairwise inequivalent irreducible unitary representations of the group $\Gamma_{1} \backslash \Gamma$; for $\vartheta \in\left(\Gamma_{1} \backslash \Gamma\right)^{*}, n_{\vartheta}$ is the dimension of $\vartheta ; Z(s, \Gamma, \vartheta)$ is the Selberg zeta-function; 1 is the trivial representation of $\Gamma_{1}$, and $U^{\mathbf{1}}$ is its induced representation to $\Gamma$.

\section{Preliminaries}

In this section we state the preliminary results that will be needed. Our main references are [Fri05a, Fri05b, [EGM98, and Ven82, and Hej76, Hej83. Unless stated otherwise, throughout this section $\Gamma<\operatorname{PSL}(2, \mathbb{C})$ is a cofinite Kleinian group and $\chi \in \operatorname{Rep}(\Gamma, V)$ is a finite-dimensional unitary representation of $\Gamma$ in $V$.

2.1. Cofinite Kleinian groups. Let $\Gamma<\operatorname{PSL}(2, \mathbb{C})$ be a cofinite Kleinian group acting on a hyperbolic three-space $\mathbb{H}^{3}$. Let $V$ be a finite-dimensional complex inner product space with inner-product $\langle,\rangle_{V}$, and let $\operatorname{Rep}(\Gamma, V)$ denote the set of finitedimensional unitary representations of $\Gamma$ in $V$. Let $\mathcal{F} \subset \Gamma$ be a fundamental domain for the action of $\Gamma$ in $\mathbb{H}^{3}$.

Let $\chi \in \operatorname{Rep}(\Gamma, V)$. The Hilbert space of $\chi$-automorphic functions is the set of measurable functions

$$
\begin{aligned}
\mathcal{H}(\Gamma, \chi) \equiv\left\{f: \mathbb{H}^{3} \rightarrow V \mid f(\gamma P)\right. & =\chi(\gamma) f(P) \forall \gamma \in \Gamma, P \in \mathbb{H}^{3}, \\
& \text { and } \left.\langle f, f\rangle \equiv \int_{\mathcal{F}}\langle f(P), f(P)\rangle_{V} d v(P)<\infty\right\} .
\end{aligned}
$$

Finally, let $\Delta=\Delta(\Gamma, \chi)$ be the corresponding positive self-adjoint Laplace-Beltrami operator on $\mathcal{H}(\Gamma, \chi)$.

Next we briefly define the concept of a singular unitary representation. Let $\mathbb{P}$ be the the boundary of $\mathbb{H}^{3}$, the Riemann sphere. For every $\zeta \in \mathbb{P}^{1}$ let $\Gamma_{\zeta}$ denote the stabilizer subgroup of $\zeta$ in $\Gamma$,

$$
\Gamma_{\zeta} \equiv\{\gamma \in \Gamma \mid \gamma \zeta=\zeta\}
$$

and let $\Gamma_{\zeta}^{\prime}$ be the maximal torsion-free parabolic subgroup of $\Gamma_{\zeta}$ (the maximal parabolic subgroup of $\Gamma_{\zeta}$ that does not contain elliptic elements). A point $\zeta \in \mathbb{P}^{1}$ is called a cusp of $\Gamma$ if $\Gamma_{\zeta}^{\prime}$ is a free abelian group of rank two. Two cusps $\zeta_{1}, \zeta_{2}$ are $\Gamma$-equivalent if $\zeta_{1} \in \Gamma \zeta_{2}$, that is, if their $\Gamma$-orbits coincide.

Every cofinite Kleinian group has finitely many equivalence classes of cusps, so we fix a set $\left\{\zeta_{\alpha}\right\}_{\alpha=1}^{\kappa(\Gamma)}$ of representatives of these equivalence classes. For notational convenience we set $\Gamma_{\alpha} \equiv \Gamma_{\zeta_{\alpha}}$ and $\Gamma_{\alpha}^{\prime} \equiv \Gamma_{\zeta_{\alpha}}^{\prime}$.

Each cusp $\zeta_{\alpha}$ has an associated lattice $\Lambda_{\alpha}$ (see [EGM98, Theorem 2.1.8]). For each cusp $\zeta_{\alpha}$ of $\Gamma$, define the singular space $V_{\alpha} \equiv\left\{v \in V \mid \chi(\gamma) v=v, \forall \gamma \in \Gamma_{\alpha}\right\}$, where $1 \leq \alpha \leq \kappa(\Gamma)$. 
A representation $\chi \in$ Rep is singular at the cusp $\zeta_{\alpha}$ of $\Gamma$ iff the subspace $V_{\alpha} \neq$ $\{0\}$. If a cusp is not singular, it is called regular.

For each cusp $\zeta_{\alpha}$, set $k_{\alpha}=\operatorname{dim}_{\mathbb{C}} V_{\alpha}$, and $k(\Gamma, \chi) \equiv \sum_{\alpha=1}^{\kappa(\Gamma)} k_{\alpha}$.

2.2. Selberg zeta-function. Let $Z(s, \Gamma, \chi)$ denote the Selberg zeta-function associated to $\Gamma$ and $\chi \in \operatorname{Rep}(\Gamma, \chi)$. We allow $\Gamma$ to be an arbitrary Kleinian group. See [Fri05a and [EGM98, Sections 5.2,5.4] for the details on its construction.

In Fri05a, we gave the meromorphic continuation of $Z(s, \Gamma, \chi)$ to the left half plane under certain technical assumptions. A practical way of understanding the zeta-function is via its logarithmic derivative. $\operatorname{For} \operatorname{Re}(s)>1$,

$$
\frac{d}{d s} \log Z(s, \Gamma, \chi)=\sum_{\{T\}_{\text {lox }}} \frac{\operatorname{tr}(\chi(T)) \log N\left(T_{0}\right)}{m(T)\left|a(T)-a(T)^{-1}\right|^{2}} N(T)^{-s} .
$$

See Fri05a for the definition of the notation used above.

Let $W(s, \Gamma, \chi)=\frac{d}{d s} \log Z(s, \Gamma, \chi)$. Then, for $\operatorname{Re} s>1$,

$$
Z(s, \Gamma, \chi)=e^{\int W(s, \Gamma, \chi) d s+C},
$$

where $C$ is chosen so

$$
\lim _{s \rightarrow \infty} Z(s, \Gamma, \chi)=1
$$

will be satisfied.

2.3. Selberg theory of $\Delta$. In this section we assume that $\Gamma$ is cofinite and we state some needed results concerning the Selberg trace formula associated to $\Gamma$ and $\chi \in$ $\operatorname{Rep}(\Gamma, V)$. We will not need the full trace formula found in Fri05a, Fri05b. Rather, only parts of its proof will be needed. More details can be found in Fri05a, Fri05b, EGM98, and [Ven82].

For $P=z+r j, P^{\prime}=z^{\prime}+r^{\prime} j \in \mathbb{H}^{3}$ set

$$
\delta\left(P, P^{\prime}\right) \equiv \cosh \left(d\left(P, P^{\prime}\right)\right)=\frac{\left|z-z^{\prime}\right|^{2}+r^{2}+r^{\prime 2}}{2 r r^{\prime}},
$$

where $d$ is the hyperbolic distance in $\mathbb{H}^{3}$.

For $k \in \mathcal{S}([1, \infty))$, a Schwartz-class function, set $K\left(P, P^{\prime}\right)=k\left(\delta\left(P, P^{\prime}\right)\right)$. Note that for any $\gamma \in \operatorname{PSL}(2, \mathbb{C})$,

$$
K\left(\gamma P, \gamma P^{\prime}\right)=K\left(P, P^{\prime}\right) \quad \text { and } \quad K\left(P, \gamma P^{\prime}\right)=K\left(\gamma^{-1} P, P^{\prime}\right) .
$$

For $\Theta \subset \Gamma, \chi \in \operatorname{Rep}(\Gamma, V)$, define

$$
K\left(P, P^{\prime}, \Theta, \psi\right) \equiv \sum_{\gamma \in \Theta} \chi(\gamma) K\left(P, \gamma P^{\prime}\right) .
$$

The series above converges absolutely and uniformly on compact subsets of $\mathbb{H}^{3} \times \mathbb{H}^{3}$.

For $\lambda \in \mathbb{C}, \lambda=1-s^{2}$, the Selberg-Harish-Chandra transform of $k, h$ is defined by

$$
h(\lambda)=h\left(1-s^{2}\right) \equiv \frac{\pi}{s} \int_{1}^{\infty} k\left(\frac{1}{2}\left(t+\frac{1}{t}\right)\right)\left(t^{s}-t^{-s}\right)\left(t-\frac{1}{t}\right) \frac{d t}{t}, \lambda=1-s^{2} .
$$

In addition, let

$$
g(x)=\frac{1}{2 \pi} \int_{\mathbb{R}} h\left(1+t^{2}\right) e^{-i t x} d t .
$$


We can start with the function $h$ and work backwards to find $k$ EGM98, Chapter 3]. The pair $h, g$ is said to be admissible if $h$ is a holomorphic function on $\{s \in \mathbb{C}|| \operatorname{Im}(s) \mid<2+\delta\}$ for some $\delta>0$, satisfying $\left.h\left(1+z^{2}\right)=O\left(1+|z|^{2}\right)^{3 / 2-\epsilon}\right)$ as $|z| \rightarrow \infty$.

For $v, w \in V$ let $v \otimes \bar{w}$ be the linear operator in $V$, defined by $v \otimes \bar{w}(x)=$ $\langle x, w\rangle v$, where $x \in V$.

Lemma 2.1 ([Fri05b]). Let $k \in \mathcal{S}([1, \infty))$ and $h: \mathbb{C} \rightarrow \mathbb{C}$ be the Selberg-HarishChandra Transform of $k$. Then

$$
\begin{aligned}
& K(P, Q, \Gamma, \chi)=\sum_{m \in \mathcal{D}} h\left(\lambda_{m}\right) e_{m}(P) \otimes \overline{e_{m}(Q)} \\
&+\frac{1}{4 \pi} \sum_{\alpha=1}^{\kappa(\Gamma)} \sum_{l=1}^{k_{\alpha}} \frac{\left[\Gamma_{\alpha}: \Gamma_{\alpha}^{\prime}\right]}{\left|\Lambda_{\alpha}\right|} \int_{\mathbb{R}} h\left(1+t^{2}\right) E_{\alpha l}(P, i t) \otimes \overline{E_{\alpha l}(Q, i t)} d t .
\end{aligned}
$$

The sum and integrals converge on compact subsets of $\mathbb{H}^{3} \times \mathbb{H}^{3}$. Here $\mathcal{D}$ is an indexing set of the eigenfunctions $e_{m}$ of $\Delta$ with corresponding eigenvalues $\lambda_{m}$, $E_{\alpha l}(P, s)$ are the Eisenstein series associated to the singular cusps of $\Gamma, k_{\alpha}=$ $\operatorname{dim}_{\mathbb{C}} V_{\alpha}$, and $\left|\Lambda_{\alpha}\right|$ is the Euclidean area of a fundamental domain for the lattice $\Lambda_{\alpha}$. If a cusp is regular, it is omitted from the sum in (2.6).

Next, we split up $K_{\Gamma}$ as a sum of two kernels. The first kernel,

$$
H_{\Gamma}(P, Q)=\frac{1}{4 \pi} \sum_{\alpha=1}^{\kappa} \sum_{l=1}^{k_{\alpha}} \frac{\left[\Gamma_{\alpha}: \Gamma_{\alpha}^{\prime}\right]}{\left|\Lambda_{\alpha}\right|} \int_{\mathbb{R}} h\left(1+t^{2}\right) E_{\alpha l}(P, i t) \otimes \overline{E_{\alpha l}(Q, i t)} d t,
$$

is not of Hilbert-Schmidt class, while the second kernel,

$$
L_{\Gamma}(P, Q)=\sum_{m \in \mathcal{D}} h\left(\lambda_{m}\right) e_{m}(P) \otimes \overline{e_{m}(Q)},
$$

is of trace class.

Suppose $Y>0$ is sufficiently large. Then for all $A>Y$, there exists a compact set $\mathcal{F}_{A} \subset \mathbb{H}^{3}$ such that

$$
\mathcal{F} \equiv \mathcal{F}_{A} \cup \mathcal{F}_{1}(A) \cup \cdots \cup \mathcal{F}_{\kappa}(A)
$$

is a fundamental domain for $\Gamma$. The sets $\mathcal{F}_{\alpha}(A)$ are cusp sectors (see [EGM98, Proposition 2.3.9). It follows that

$$
\begin{aligned}
& \lim _{A \rightarrow \infty}\left(\int_{\mathcal{F}_{A}} \operatorname{tr}_{V}\left(K_{\Gamma}(P, P)\right)\right.\left.d v(P)-\int_{\mathcal{F}_{A}} \operatorname{tr}_{V}\left(H_{\Gamma}(P, P)\right) d v(P)\right) \\
&=\int_{\mathcal{F}} \operatorname{tr}_{V}\left(L_{\Gamma}(P, P)\right) d v(P)=\sum_{m \in \mathcal{D}} h\left(\lambda_{m}\right)<\infty .
\end{aligned}
$$

The infinite sum is absolutely convergent.

Let $\mathfrak{S}(s)$ denote the automorphic scattering matrix associated to $\Gamma$ and $\chi$ (see Fri05b), and let

$$
\phi(s)=\operatorname{det} \mathfrak{S}(s) .
$$


Upon applying the vector form of the Maaß-Selberg relations (see [Roe66, Ven82, Fri05b]), we obtain

$$
\begin{aligned}
& \int_{\mathcal{F}_{A}} \operatorname{tr}_{V}\left(H_{\Gamma}(P, P)\right) d v(P) \\
& \quad=g(0) k(\Gamma, \chi) \log (A)-\frac{1}{4 \pi} \int_{\mathbb{R}} \frac{\phi^{\prime}}{\phi}(i t) h\left(1+t^{2}\right) d t+\frac{h(1) \operatorname{tr} \mathfrak{S}(0)}{4}+\underset{A \rightarrow \infty}{o(1)} .
\end{aligned}
$$

The integral on the right-hand side converges absolutely.

\section{INDUCED REPRESENTATIONS}

Let $\Gamma$ be an arbitrary Kleinian group, and let $\Gamma_{1} \triangleleft \Gamma$ be a finite-index normal subgroup of index $n$. Let $\mathcal{F}, \mathfrak{F}$ be fundamental domains of $\Gamma, \Gamma_{1}$ respectively, with $\left[\alpha_{i}\right]_{i=1}^{n}$ a complete set of representatives for the right-cosets of $\Gamma_{1} \backslash \Gamma$, satisfying

$$
\mathfrak{F}=\bigcup_{i=1}^{n} \alpha_{i}(\mathcal{F})
$$

Let $V$ be a finite-dimensional hermitian vector space, and let $\chi$ be a finitedimensional unitary representation of $\Gamma_{1}$ in $V$. Set

$$
\bar{\chi}(\gamma)=\left\{\begin{array}{cc}
\chi(\gamma), & \gamma \in \Gamma_{1} \\
0, & \gamma \notin \Gamma_{1}
\end{array}\right.
$$

Let $\psi \equiv U^{\chi}$ be the induced representation of $\chi$ from $\Gamma_{1}$ to $\Gamma$. More explicitly,

$$
\psi: \Gamma \mapsto \operatorname{GL}\left(V^{n}\right),
$$

and for $\gamma \in \Gamma$ and $v_{i} \in V$,

$$
\psi(\gamma)\left(\sum_{i=1}^{n} \oplus v_{i}\right)=\sum_{i=1}^{n} \oplus \sum_{j=1}^{n} \bar{\chi}\left(\alpha_{i} \gamma \alpha_{j}^{-1}\right) v_{j} .
$$

It follows from (3.2) that for $\gamma \in \Gamma$,

$$
\operatorname{tr}_{V^{n}} \psi(\gamma)=\sum_{i=1}^{n} \operatorname{tr}_{V} \bar{\chi}\left(\alpha_{i} \gamma \alpha_{i}^{-1}\right)
$$

We will need the following result (see [RS71] and [VZ83, Theorem 2.1]):

Lemma 3.1. There exists an isometry between the Hilbert spaces $\mathcal{H}\left(\Gamma_{1}, \chi\right)$ and $\mathcal{H}(\Gamma, \psi)$, which takes the operator $\Delta\left(\Gamma_{1}, \chi\right)$ to $\Delta(\Gamma, \psi)$.

We abuse notation and call a set $\Theta \subset \Gamma$ normal if for all $\gamma \in \Gamma$, we have $\gamma \Theta \gamma^{-1}=\Theta$.

Lemma 3.2. Let $\Gamma_{1} \triangleleft \Gamma$ be a finite-index, normal subgroup of $\Gamma$ of index $n$, $\chi \in \operatorname{Rep}\left(\Gamma_{1}, V\right), \psi \equiv U^{\chi} \in \operatorname{Rep}\left(\Gamma, V^{n}\right)$, and let $\Theta \subset \Gamma$ be a normal subset. Suppose that

$$
\int_{\mathcal{F}} \operatorname{tr}_{V^{n}} K(P, P, \Theta, \psi) d v(P)
$$

converges absolutely. Then

$$
\int_{\mathcal{F}} \operatorname{tr}_{V^{n}} K(P, P, \Theta, \psi) d v(P)=\int_{\mathfrak{F}} \operatorname{tr}_{V} K\left(P, P, \Theta \cap \Gamma_{1}, \chi\right) d v(P) .
$$


Proof. By (3.3),

$$
\begin{aligned}
& \int_{\mathcal{F}} \operatorname{tr}_{V^{n}} K(P, P, \Theta, \psi) d v(P) \\
& =\sum_{i=1}^{n} \int_{\mathcal{F}} \sum_{\gamma \in \Theta} \operatorname{tr}_{V} \bar{\chi}\left(\alpha_{i} \gamma \alpha_{i}^{-1}\right) K(P, \gamma P) d v(P) \\
& =\sum_{i=1}^{n} \int_{\mathcal{F}} \sum_{\substack{\gamma \in \Theta \\
\alpha_{i} \gamma \alpha_{i}^{-1} \in \Gamma_{1}}} \operatorname{tr}_{V} \chi\left(\alpha_{i} \gamma \alpha_{i}^{-1}\right) K(P, \gamma P) d v(P) \\
& =\sum_{i=1}^{n} \int_{\mathcal{F}} \sum_{\gamma \in \Theta \cap \Gamma_{1}} \operatorname{tr}_{V} \chi\left(\alpha_{i} \gamma \alpha_{i}^{-1}\right) K(P, \gamma P) d v(P) \quad \text { (by normality). }
\end{aligned}
$$

Now, since $\Theta$ and $\Gamma_{1}$ are normal, $\gamma \in \Theta \cap \Gamma_{1}$ implies that $\alpha_{i} \gamma \alpha_{i}^{-1} \in \Theta \cap \Gamma_{1}$. Also, as $\gamma$ goes through each element of $\Theta \cap \Gamma_{1}$, so does $\alpha_{i} \gamma \alpha_{i}^{-1}$. So, for each $i$, setting $\beta=\alpha_{i} \gamma \alpha_{i}^{-1}$ we obtain

$$
\begin{aligned}
& \sum_{i=1}^{n} \int_{\mathcal{F}} \sum_{\gamma \in \Theta \cap \Gamma_{1}} \operatorname{tr}_{V} \chi\left(\alpha_{i} \gamma \alpha_{i}^{-1}\right) K(P, \gamma P) d v(P) \\
& \quad=\sum_{i=1}^{n} \int_{\mathcal{F}} \sum_{\beta \in \Theta \cap \Gamma_{1}} \operatorname{tr}_{V} \chi(\beta) K\left(P, \alpha_{i}^{-1} \beta \alpha_{i} P\right) d v(P) \\
& \quad=\sum_{i=1}^{n} \int_{\mathcal{F}} \sum_{\beta \in \Theta \cap \Gamma_{1}} \operatorname{tr}_{V} \chi(\beta) K\left(\alpha_{i} P, \beta \alpha_{i} P\right) d v(P) \\
& =\sum_{i=1}^{n} \int_{\alpha_{i}(\mathcal{F})} \sum_{\beta \in \Theta \cap \Gamma_{1}} \operatorname{tr}_{V} \chi(\beta) K(Q, \beta Q) d v(Q) \\
& =\int_{\mathfrak{F}_{\beta \in \Theta \cap \Gamma_{1}}} \sum_{\operatorname{tr}_{V} \chi(\beta) K(Q, \beta Q) d v(Q)} \\
& =\int_{\mathfrak{F}} K\left(P, P, \Theta \cap \Gamma_{1}, \chi\right) d v(P),
\end{aligned}
$$

where in (3.5) we set $Q=\alpha_{i} P$. In (3.4) we used Equation (2.3), and we used the fact that $d v\left(\alpha_{i} Q\right)=d v(Q)$; and in (3.6) we tiled the fundamental domain $\mathcal{F}$ according to (3.1).

Lemma 3.2 works well when the point-pair-invariant $k$ gives rise to an integral kernal of trace-class. However, a careful look at the proof shows that we could replace $\mathcal{F}$ by a truncated fundamental domain $\mathcal{F}_{A}$.

For the rest of this section, we assume that $\Gamma$ is cofinite. Let $Y>0$ be sufficiently large so that for $A>Y, \mathcal{F}$ decomposes into $\mathcal{F}=\mathcal{F}_{A} \cup \mathcal{F}^{A}$, where $\mathcal{F}_{A}$ is compact and $\mathcal{F}^{A}$ is a union of cusp sectors (2.7). Since

$$
\mathfrak{F}=\bigcup_{i=1}^{n} \alpha_{i}(\mathcal{F})
$$

it follows that $\mathfrak{F}=\mathfrak{F}_{A} \cup \mathfrak{F}^{A}$ with $\mathfrak{F}_{A}=\bigcup_{i=1}^{n} \alpha_{i}\left(\mathcal{F}_{A}\right)$ and $\mathfrak{F}^{A}=\bigcup_{i=1}^{n} \alpha_{i}\left(\mathcal{F}^{A}\right) ; \mathfrak{F}^{A}$ is also a union of cusp sectors. 
Lemma 3.3. Let $\Gamma_{1} \triangleleft \Gamma$ be a finite-index, normal subgroup of $\Gamma$ of index $n$, $\chi \in \operatorname{Rep}\left(\Gamma_{1}, V\right), \psi \equiv U^{\chi} \in \operatorname{Rep}\left(\Gamma, V^{n}\right)$, and let $\Theta \subset \Gamma$ be a normal subset. Suppose that

converges absolutely. Then

$$
\int_{\mathcal{F}_{A}} \operatorname{tr}_{V^{n}} K(P, P, \Theta, \psi) d v(P)
$$

$$
\int_{\mathcal{F}_{A}} \operatorname{tr}_{V^{n}} K(P, P, \Theta, \psi) d v(P)=\int_{\mathfrak{F}_{A}} \operatorname{tr}_{V} K\left(P, P, \Theta \cap \Gamma_{1}, \chi\right) d v(P) .
$$

\section{Factorization formula of the Selberg zeta-function}

In this section we prove the analogue of the Venkov-Zograf factorization formula ([VZ83] ) for arbitrary Kleinian groups.

Throughout this section, $\Gamma$ is an arbitrary Kleinian group and $\Gamma_{1} \triangleleft \Gamma$ is a finiteindex normal subgroup of index $n$.

Theorem 4.1. Suppose that $\Gamma_{1} \triangleleft \Gamma, \chi \in \operatorname{Rep}\left(\Gamma_{1}, V\right)$, and that $\psi=U^{\chi} \in$ $\operatorname{Rep}\left(\Gamma, V^{n}\right)$. Then for $\operatorname{Re}(s)>1$,

$$
Z\left(s, \Gamma_{1}, \chi\right)=Z(s, \Gamma, \psi) .
$$

Proof. By (2.2), it suffices to show that

$$
W\left(s, \Gamma_{1}, \chi\right)=W(s, \Gamma, \psi) .
$$

Let $\Theta=\Gamma^{\text {lox }} \subset \Gamma$ be the set of all loxodromic elements of $\Gamma$. Note that $\Theta$ is normal since the conjugate of a loxodromic element is loxodromic. For $\operatorname{Re} s>1, \delta>1$, set

$$
k_{s}(\delta) \equiv \frac{1}{4 \pi} \frac{\left(\delta+\sqrt{\delta^{2}-1}\right)^{-s}}{\sqrt{\delta^{2}-1}} .
$$

For $P, P^{\prime} \in \mathbb{H}^{3}$, set $K_{s}\left(P, P^{\prime}\right)=k_{s}\left(\delta\left(P, P^{\prime}\right)\right)$, and, using (2.4), define $K_{s}\left(P, P^{\prime}, \Theta, \psi\right)$ and $K_{s}\left(P, P^{\prime}, \Theta \cap \Gamma_{1}, \chi\right)$. By [EGM98, pages 185-198] and [Fri05a, Lemma 6.3], it follows that

$$
W\left(s, \Gamma_{1}, \chi\right)=\int_{\mathfrak{F}} \operatorname{tr}_{V} K_{s}\left(P, P, \Theta \cap \Gamma_{1}, \chi\right) d v(P)
$$

and

$$
W(s, \Gamma, \psi)=\int_{\mathcal{F}} \operatorname{tr}_{V^{n}} K_{s}(P, P, \Theta, \psi) d v(P) .
$$

The result now follows from Lemma 3.2

Compare our proof with the proof given in VZ83, Theorem 3.1].

If we let $\chi=1$, the trivial one-dimensional representation, it follows that $\psi=U^{1}$ can be decomposed into irreducible sub-representations, explicitly:

$$
\psi=\bigoplus_{\vartheta \in\left(\Gamma_{1} \backslash \Gamma\right)^{*}} n_{\vartheta} \vartheta
$$

where $\left(\Gamma_{1} \backslash \Gamma\right)^{*}$ denotes the set of all pairwise inequivalent irreducible unitary representations of the groun $11 \Gamma_{1} \backslash \Gamma$, and $n_{\vartheta}$ is the dimension of $\vartheta$. For more details see [ir76].

Next, by (2.1) and (2.2) it follows that for $\vartheta_{1}, \vartheta_{2} \in \operatorname{Rep}\left(\Gamma, V_{i}\right)(i=1,2)$,

$$
Z\left(s, \Gamma, \vartheta_{1} \oplus \vartheta_{2}\right)=Z\left(s, \Gamma, \vartheta_{1}\right) Z\left(s, \Gamma, \vartheta_{2}\right) .
$$

\footnotetext{
${ }^{1}$ Of course, each $\chi$ must be extended from $\Gamma_{1} \backslash \Gamma$ to $\Gamma$.
} 
We have

Theorem 4.2. Let $\Gamma_{1} \triangleleft \Gamma$. Then for $\operatorname{Re} s>1$,

$$
Z\left(s, \Gamma_{1}, \mathbf{1}\right)=Z\left(s, \Gamma, U^{\mathbf{1}}\right)=\prod_{\vartheta \in\left(\Gamma_{1} \backslash \Gamma\right)^{*}} Z(s, \Gamma, \vartheta)^{n_{\vartheta}} .
$$

\section{FACTORIZATION FORMUla FOR THE DETERMinant OF THE SCATTERING MATRIX}

In this section we prove the analogous result to Theorem 4.1 for the automorphic scattering matrix. Throughout this section, $\Gamma$ is a cofinite Kleinian group.

Let $\Gamma_{1} \triangleleft \Gamma, \chi \in \operatorname{Rep}\left(\Gamma_{1}, V\right)$, and $\psi=U^{\chi}$. Let $k \in \mathcal{S}([1, \infty))$, and let $h$ be given by (2.5). It follows form Lemma 3.1 that $\Delta\left(\Gamma_{1}, \chi\right)$ and $\Delta(\Gamma, \psi)$ have the same

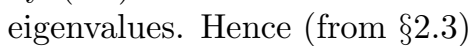

$$
\int_{\mathcal{F}} \operatorname{tr}_{V^{n}} L(P, P, \Gamma, \psi) d v(P)=\int_{\mathfrak{F}} \operatorname{tr}_{V} L\left(P, P, \Gamma_{1}, \chi\right) d v(P)=\sum_{m \in \mathcal{D}} h\left(\lambda_{m}\right) .
$$

Now, since $\int_{\mathcal{F}_{A}} \operatorname{tr}_{V^{n}} K(P, P, \Gamma, \psi) d v(P)=\int_{\mathfrak{F}_{A}} \operatorname{tr}_{V} K\left(P, P, \Gamma_{1}, \chi\right) d v(P)$, it follows from Equation (2.8) that

$$
\int_{\mathcal{F}_{A}} \operatorname{tr}_{V^{n}} H(P, P, \Gamma, \psi) d v(P)=\int_{\mathfrak{F}_{A}} \operatorname{tr}_{V} H\left(P, P, \Gamma_{1}, \chi\right) d v(P)+\underset{A \rightarrow \infty}{o(1)} .
$$

However, from Equation (2.9), we obtain

$$
\begin{aligned}
& \int_{\mathcal{F}_{A}} \operatorname{tr}_{V^{n}} H(P, P, \Gamma, \psi) d v(P) \\
& =g(0) k(\Gamma, \psi) \log (A)-\frac{1}{4 \pi} \int_{\mathbb{R}} \frac{\phi_{\Gamma}^{\prime}}{\phi_{\Gamma}}(i t) h\left(1+t^{2}\right) d t+\frac{h(1) \operatorname{tr} \mathfrak{S}_{\Gamma}(0)}{4}+\underset{A \rightarrow \infty}{o(1)}
\end{aligned}
$$

and

$$
\begin{aligned}
& \int_{\mathcal{F}_{A}} \operatorname{tr}_{V} H\left(P, P, \Gamma_{1}, \chi\right) d v(P) \\
= & g(0) k\left(\Gamma_{1}, \chi\right) \log (A)-\frac{1}{4 \pi} \int_{\mathbb{R}} \frac{\phi_{\Gamma_{1}}^{\prime}}{\phi_{\Gamma_{1}}}(i t) h\left(1+t^{2}\right) d t+\frac{h(1) \operatorname{tr} \mathfrak{S}_{\Gamma_{1}}(0)}{4}+\underset{A \rightarrow \infty}{o(1)} .
\end{aligned}
$$

Hence, we have

$$
\begin{aligned}
& g(0) k(\Gamma, \psi) \log (A)-\frac{1}{4 \pi} \int_{\mathbb{R}} \frac{\phi_{\Gamma}^{\prime}}{\phi_{\Gamma}}(i t) h\left(1+t^{2}\right) d t+\frac{h(1) \operatorname{tr} \mathfrak{S}_{\Gamma}(0)}{4} \\
= & g(0) k\left(\Gamma_{1}, \chi\right) \log (A)-\frac{1}{4 \pi} \int_{\mathbb{R}} \frac{\phi_{\Gamma_{1}}^{\prime}}{\phi_{\Gamma_{1}}}(i t) h\left(1+t^{2}\right) d t+\frac{h(1) \operatorname{tr} \mathfrak{S}_{\Gamma_{1}}(0)}{4}+\underset{A \rightarrow \infty}{o(1)} .
\end{aligned}
$$

Equation (5.3) is true for all admissible pairs $h, g$. Hence we must have

$$
\begin{aligned}
k(\Gamma, \psi) & =k\left(\Gamma_{1}, \chi\right), \\
\operatorname{tr} \mathfrak{S}_{\Gamma}(0) & =\operatorname{tr} \mathfrak{S}_{\Gamma_{1}}(0),
\end{aligned}
$$

and

$$
\frac{\phi_{\Gamma}^{\prime}}{\phi_{\Gamma}}(z)=\frac{\phi_{\Gamma_{1}}^{\prime}}{\phi_{\Gamma_{1}}}(z)
$$

We will prove (5.4) shortly. 
Theorem 5.1. Let $\Gamma_{1} \triangleleft \Gamma, \chi \in \operatorname{Rep}\left(\Gamma_{1}, V\right)$, and $\psi=U^{\chi} \in \operatorname{Rep}\left(\Gamma, V^{n}\right)$. Let

$$
\begin{gathered}
\phi_{\Gamma_{1}}(s) \equiv \operatorname{det} \mathfrak{S}_{\Gamma_{1}}(s) \equiv \operatorname{det} \mathfrak{S}\left(s, \Gamma_{1}, \chi\right), \\
\phi_{\Gamma}(s) \equiv \operatorname{det} \mathfrak{S}_{\Gamma}(s) \equiv \operatorname{det} \mathfrak{S}(s, \Gamma, \psi) .
\end{gathered}
$$

Then for all regular $s$,

$$
\phi_{\Gamma_{1}}(s)=\phi_{\Gamma}(s) .
$$

Proof. For $r>0, z \in \mathbb{C}$, let $h(z)=e^{-r z^{2}}$ (and it follows that)

$$
g(x)=\frac{e^{-r}}{\sqrt{4 \pi r}} e^{-x^{2} /(4 r)} .
$$

By considering the asymptotics of (5.3), it follows that

$$
k(\Gamma, \psi)=k\left(\Gamma_{1}, \chi\right)
$$

and

$$
\operatorname{tr} \mathfrak{S}_{\Gamma}(0)=\operatorname{tr} \mathfrak{S}_{\Gamma_{1}}(0)
$$

Hence

$$
\int_{\mathbb{R}} \frac{\phi_{\Gamma}^{\prime}}{\phi_{\Gamma}}(i t) h\left(1+t^{2}\right) d t=\int_{\mathbb{R}} \frac{\phi_{\Gamma_{1}}^{\prime}}{\phi_{\Gamma_{1}}}(i t) h\left(1+t^{2}\right) d t,
$$

which implies that

$$
\int_{\mathbb{R}} \frac{\phi_{\Gamma}^{\prime}}{\phi_{\Gamma}}(i t) e^{-r t^{2}} d t=\int_{\mathbb{R}} \frac{\phi_{\Gamma_{1}}^{\prime}}{\phi_{\Gamma_{1}}}(i t) e^{-r t^{2}} d t \quad(r>0) .
$$

Now, by the functional equation for $\mathfrak{S}_{\Gamma}(s)$ (see [Fri05a]

$$
\mathfrak{S}_{\Gamma}(s) \mathfrak{S}_{\Gamma}(-s)=I \text {. }
$$

So

$$
\phi_{\Gamma}(s) \phi_{\Gamma}(-s)=1 .
$$

Hence $\frac{\phi_{\Gamma}^{\prime}}{\phi_{\Gamma}}(i t)$ is an even function of $t$; so is $\frac{\phi_{\Gamma_{1}}^{\prime}}{\phi_{\Gamma_{1}}}(i t)$. Thus

$$
\int_{0}^{\infty} \frac{\phi_{\Gamma}^{\prime}}{\phi_{\Gamma}}(i t) e^{-r t^{2}} d t=\int_{0}^{\infty} \frac{\phi_{\Gamma_{1}}^{\prime}}{\phi_{\Gamma_{1}}}(i t) e^{-r t^{2}} d t \quad(r>0) .
$$

Next, the substitution $u=t^{2}$ allows us to rewrite the above integral as a Laplace transform, and by uniqueness (the Laplace transform is invertible), it follows that $\frac{\phi_{\Gamma}^{\prime}}{\phi_{\Gamma}}(i t)=\frac{\phi_{\Gamma_{1}}^{\prime}}{\phi_{\Gamma_{1}}}(i t)$, and (by analytic continuation)

$$
\frac{\phi_{\Gamma}^{\prime}}{\phi_{\Gamma}}(s)=\frac{\phi_{\Gamma_{1}}^{\prime}}{\phi_{\Gamma_{1}}}(s) .
$$

Integrating and exponentiating give us a constant $C_{1}$ so that

$$
\phi_{\Gamma}=C_{1} \cdot \phi_{\Gamma_{1}} .
$$

However, the functional equation (for $\mathfrak{S}_{\Gamma}(s)$ ) implies that

$$
\phi_{\Gamma}(0)=(-1)^{\left(k_{\Gamma}-\operatorname{tr} \mathfrak{S}_{\Gamma}(0)\right) / 2}=(-1)^{\left(k_{\Gamma_{1}}-\operatorname{tr} \mathfrak{S}_{\Gamma_{1}}(0)\right) / 2}=\phi_{\Gamma_{1}}(0) \neq 0 .
$$

Hence $C_{1}=1$. 


\section{ACKNOWLEDGEMENTS}

I would like to thank Professor Leon Takhtajan for originally suggesting this problem to me and for reading this paper. I would also like to thank Peter Zograf for reading this paper and for useful discussions, and the anonymous referee for correcting some errors.

\section{REFERENCES}

[EGM98] J. Elstrodt, F. Grunewald, and J. Mennicke, Groups acting on hyperbolic space, Harmonic analysis and number theory, Springer Monographs in Mathematics, SpringerVerlag, Berlin, 1998. MR1483315 (98g:11058)

[Fri05a] Joshua S. Friedman, The Selberg trace formula and Selberg zeta-function for cofinite Kleinian groups with finite-dimensional unitary representations, Math. Z. 250 (2005), no. 4, 939-965. MR 2180383 (2006g:11099)

[Fri05b] - The Selberg trace formula and Selberg zeta-function for cofinite Kleinian groups with finite-dimensional unitary representations. Ph.D. Thesis, Stony Brook University, 2005, http://arxiv.org/abs/math. NT/0612807.

[Hej76] Dennis A. Hejhal, The Selberg trace formula for PSL(2, R). Vol. I, Lecture Notes in Mathematics, vol. 548, Springer-Verlag, Berlin, 1976. MR0439755 (55:12641)

[Hej83] , The Selberg trace formula for $\operatorname{PSL}(2, \mathbf{R})$. Vol. 2, Lecture Notes in Mathematics, vol. 1001, Springer-Verlag, Berlin, 1983. MR0711197(86e:11040)

[Kir76] A. A. Kirillov, Elements of the theory of representations, Springer-Verlag, Berlin, 1976, Translated from the Russian by Edwin Hewitt, Grundlehren der Mathematischen Wissenschaften, Band 220. MR0412321 (54:447)

[Roe66] Walter Roelcke, Das Eigenwertproblem der automorphen Formen in der hyperbolischen Ebene. I, II, Math. Ann. 167 (1966), 292-337; ibid. 168 (1966), 261-324. MR 1513277

[RS71] D. B. Ray and I. M. Singer, R-torsion and the Laplacian on Riemannian manifolds, Advances in Math. 7 (1971), 145-210. MR0295381 (45:4447)

[Ven82] A. B. Venkov, Spectral theory of automorphic functions, Proc. Steklov Inst. Math. 1982, no. 4(153) (1983); a translation of Trudy Mat. Inst. Steklov. 153 (1981). MR665585 (85j:11060a), MR0692019 (85j:11060b)

[VZ83] A. B. Venkov and P. G. Zograf, Analogues of Artin's factorization formulas in the spectral theory of automorphic functions associated with induced representations of Fuchsian groups, Izv. Akad. Nauk SSSR Ser. Mat. 46 (1982), 1150-1158, 1343; translated in Math. USSR-Izvestiya 21 (1983), no. 3, 435-443. MR0682487 (85f:11041)

Department of Mathematics and Sciences, United States Merchant Marine Academy, 300 Steamboat Road, Kings Point, New York 11024

E-mail address: CrownEagle@gmail.com, friedmanj@usmma.edu, joshua@math.sunysb.edu 